JOURNAL OF RESEARCH of the National Bureau of Standards - B. Mathematics and Mathematical Physics Vol. 71B, Nos 2 \& 3, April-September 1967

\title{
E-Transforms (II) *
}

\section{F. M. Ragab**}

(April 20, 1967)

The following class of integral transform pairs is established

$$
\begin{gathered}
g(x)=\int_{0}^{\infty} E\left(\begin{array}{l}
\left.\nu-i x, \nu+i x, \alpha_{1}, \ldots, \alpha_{p}: \frac{1}{y}\right) f(y) d y, \\
\beta_{1}, \ldots . \beta_{q}
\end{array}\right) \\
f(x)=\frac{x^{\nu-1}}{i \pi^{2}} \int_{0}^{\infty} y g(y)\left[\frac{1}{i} \sum_{i,-i} i x^{i y} \sin (i y+\nu) \pi E\left(\begin{array}{l}
1-\nu-i y, \beta_{1}-\nu-i y, \ldots ., \beta_{q}-\nu-i y: x \\
1-2 i y, \alpha_{1}-\nu-i y, . ., \alpha_{p}-\nu-i y
\end{array}\right)\right] d y .
\end{gathered}
$$

The kernel in the transform (1) is MacRobert's E-function and integration is performed with respect to the argument of this function. In the inversion formula (2), the kernel is likewise an $E$-function, but the integration is performed with respect to its parameters.

Known special cases of this general transform pair is the Kantorovich-Lebedev transforms pair:

$$
\begin{gathered}
g(x)=\frac{2}{\pi^{2}} x \sinh (\pi x) \int_{0}^{\infty} y^{-1} K_{i x}(y) f(y) d y, \\
f(x)=\int_{0}^{\infty} K_{i y}(x) g(y) d y,
\end{gathered}
$$

and the generalized Mehler transform pair

$$
\begin{gathered}
g(x)=\frac{x}{\pi} \sinh (\pi x) \Gamma\left(\frac{1}{2}-k+i x\right) \Gamma\left(\frac{1}{2}-k-i x\right) \int_{0}^{\infty} P_{i x-1 / 2}^{k}(y) f(y) d y, \\
f(x)=\int_{0}^{\infty} P_{i y-1 / 2}^{k}(x) g(y) d y .
\end{gathered}
$$

Key Words: $E$-functions, integral transforms, inversion formulas, kernels.

\section{Introduction}

In this paper we establish the following class of integral transforms:

$$
\begin{aligned}
& g(x)=\int_{0}^{\infty} E\left(\begin{array}{l}
\nu-i x, \nu+i x, \alpha_{1}, \ldots, \alpha_{p}: \frac{1}{y} \\
\beta_{1}, \ldots, \beta_{q}
\end{array}\right) f(y) d y \\
& f(x)=\frac{x^{\nu-1}}{i \pi^{2}} \int_{0}^{x} y g(y)\left[\frac{1}{i} \sum_{i,-i} i x^{i y} \sin (i y+\nu) \pi E\left(\begin{array}{l}
1-\nu-i y, \beta_{1}-\nu-i y, . . ., \beta_{q}-\nu-i y: x \\
1-2 i y, \alpha_{1}-\nu-i y, . . ., \alpha_{p}-\nu-i y
\end{array}\right)\right] d y
\end{aligned}
$$

where the integrals are convergent and the symbol $\sum_{i,-i}$ means that in the expression following it $i$ is to be replaced by $-i$ and the two expressions are to be added.

The kernel in the transform (1) is MacRobert's $E$-function whose definitions and properties

*An invited paper. (Sponsored by the Mathematics Research Center, United States Army, Madison, Wisconsin, under Contract No.: DA-11-022-ORD-2059.) **Present address: Faculty of Engineering, Cairo University, Cairo, Eqypt, United Arab Republic. 
are given in $[1]^{1}$ pp. 348-358, and which will be discussed further in section 2 . The integration in this transform is performed with respect to the argument of the $E$-function. In the inversion formula (2), the kernel is likewise an $E$-function, but the integration is performed with respect to its parameters. Known special cases of our general transform pair are the Kantorovich-Lebedev transform pair (see [2], pp. 175-177: [3], pp. 229-241 and [4], pp. 33-40)

$$
\begin{gathered}
g(x)=\frac{2}{\pi^{2}} x \sinh \pi x \int_{0}^{\infty} y^{-1} K_{i x}(y) f(y) d y, \\
f(x)=\int_{0}^{\infty} K_{i y}(x) g(y) d y ;
\end{gathered}
$$

and the generalized Mehler transform pair (see [5] pp. 57-59 and [6]).

$$
\begin{aligned}
& g(x)=\frac{x}{\pi} \sinh \pi x \Gamma\left(\frac{1}{2}-k+i x\right) \Gamma\left(\frac{1}{2}-k-i x\right) \int_{1}^{\infty} P_{i x-\frac{1}{2}}^{k}(y) f(y) d y \\
& f(x)=\int_{0}^{\infty} P_{i y-\frac{1}{2}}^{k}(x) g(y) d y .
\end{aligned}
$$

Section 2 contains a treatment of the $E$-function and our main transform pair is derived in section 3. Section 4 contains the derivation of the Kantorovich-Lebedev and Mehler transforms and other new integral transforms.

The Mellin transform ([7], p. 7)

$$
g(s)=\int_{0}^{\infty} x^{s-1} f(x) d y
$$

and its inversion formula

$$
f(x)=\frac{1}{2 \pi i} \int_{c-i \infty}^{c+\infty} x^{-s} g(s) d s
$$

will be utilized in the proofs.

Also the following formulas are required in the proofs: ([1], p. 374):

$$
E\left(p: \alpha_{r}: q: \rho_{t}: z\right)=\frac{1}{2 \pi i} \int \frac{\Gamma(\zeta) \prod_{r=1}^{p} \Gamma\left(\alpha_{r}-\zeta\right)}{\prod_{t=1}^{q} \Gamma\left(\rho_{t}-\zeta\right)} z^{\zeta} d \zeta
$$

where $|\operatorname{amp} z|<\frac{1}{2}(p-q+1) \pi$ and the contour of integration is of Barnes's type with loops, if necessary, to separate the pole at the origin from the poles at $\alpha_{1}, \alpha_{2}, \ldots ., \alpha_{p}$ : ([1], p. 257):

$$
K_{n}(z)=\frac{\pi}{2 \sin n \pi}\left\{I_{-n}(z)-I_{n}(z)\right\}:
$$

[1], p. 347:

$$
I_{n}(z)=\frac{1}{\Gamma(n+1)}\left(\frac{1}{2} z\right)^{n} e^{-z} F\left(\begin{array}{c}
n+\frac{1}{2}: 2 z \\
2 n+1
\end{array}\right)
$$

'Figures in brachets indicate the literature references at the end of this paper. 
and [1], p. 262:

$$
F\left(\begin{array}{l}
-n, n+1 ;-z \\
1-m
\end{array}\right)=\Gamma(1-m)\left(\frac{z}{1+z}\right)^{\frac{1}{2} m} P_{n}^{m}(2 \mathrm{z}+1)
$$

\section{Properties of the E-Function}

If $p \leqslant q$ then the $E$-function is defined as

$$
E\left(p ; \alpha_{r}: q ; \rho_{t}: z\right)=\frac{\Gamma\left(\alpha_{1}\right) \ldots \Gamma\left(\alpha_{p}\right)}{\Gamma\left(\rho_{1}\right) \ldots \Gamma\left(\rho_{q}\right)} F\left(p ; \alpha_{r}: q ; \rho_{t}:-\frac{1}{z}\right) .
$$

When $p \geqslant q+1,|\arg z|<\pi$, then the $E$-function (see [1], p. 353) can be shown to be

$$
\begin{aligned}
E\left(p ; \alpha_{r}: q ; \rho_{t}: z\right)=\sum_{r=1}^{p} \prod_{r=1}^{p} \Gamma\left(\alpha_{s}-\right. & \left.\alpha_{r}\right)\left\{\prod_{t=1}^{q} \Gamma\left(\rho_{t}-\alpha_{r}\right)\right\}^{-1} \Gamma\left(\alpha_{r}\right) Z^{\alpha_{r}} \\
& \times{ }_{q+1} F_{p-1}\left(\begin{array}{c}
\alpha_{r}, \alpha_{r}-\rho_{1}+1, \ldots ., \alpha_{r}-p_{q}+1 ;(-1)^{p-q} z \\
\alpha_{r}-\alpha_{1}+1, \ldots . . . ., \alpha_{r}-\alpha_{p}+1
\end{array}\right),
\end{aligned}
$$

where the asterisk means that the factor $\alpha_{r}-\alpha_{r}+1$ is omitted. To familarize ourselves with the $E$-function, the following relations may be worth noting: From the definition (13) it is clear that the - $E$-function is immediately related to the generalized hypergeometric function

$$
{ }_{p} F_{q}\left(\begin{array}{l}
\alpha_{r}: z \\
\rho_{t}
\end{array}\right)=\sum_{n=0}^{\infty} \frac{\left(\alpha_{1}: n\right) \ldots\left(\alpha_{p} ; n\right)}{(1 ; n)\left(\rho_{1}: n\right) \ldots\left(\rho_{q} ; n\right)} z^{n}
$$

and reduces to single expressions in the ordinary or Gauss hypergeometric function when $p=2$, $q=1$. For $p=1, q=1$ it is evident that the $E$-function reduces to the confluent hypergeometric function or Kummer's function. The case $p=1, q=0$ yields the relation

$$
E(\alpha:: z)=\Gamma(\alpha)(1+1 / z)^{-\alpha}
$$

The case $p=0, q=1$ gives the relation

$$
E(: \nu+1:: z)=z^{1 / \frac{\nu}{2}} I_{\nu}\left(2 z^{-1 / 2}\right)
$$

The case $p=2, q=0$ yields the relations (see [1], p. 351)

$$
\begin{gathered}
\cos n \pi E\left(\frac{1}{2}+n, \frac{1}{2}-n:: 2 z\right)=(2 \pi z)^{\frac{1}{2} z} e^{z} K_{n}(z), \\
E\left(\frac{1}{2}-k+m, \frac{1}{2}-k-m:: z\right)=\Gamma\left(\frac{1}{2}-k-m\right) \Gamma\left(\frac{1}{2}-k+m\right) z^{-k} e^{\frac{1}{2} z} W_{k, m}(z),
\end{gathered}
$$

where $K_{n}(z)$ and $W_{k, m}(z)$ are the modified Bessel function and Whittaker function respectively. Also it is evident from the definitions of the $E$-function that for $p=q=0$

$$
E(:: z)=\exp (-1 / z) \text {. }
$$

More complicated parameters in the $E$-function lead to the equivalence of the $E$-function with products of Whittaker functions, Hankel functions, Lommel functions and other special functions. Some examples are

$$
\begin{aligned}
W_{k, m}(2 i z) W_{k, m}(-2 i z)=\pi^{-1 / 2}\left(\frac{z}{2}\right)^{2 k}\{ & \left.\Gamma\left(\frac{1}{2}-k+m\right) \Gamma\left(\frac{1}{2}-k-m\right)\right\}^{-1} \\
& \times E\left(\frac{1}{2}-k+m, \frac{1}{2}-k-m, \frac{1}{2}-k, 1-k: 1-2 k: \frac{z^{2}}{4}\right),
\end{aligned}
$$




$$
\begin{gathered}
e^{-\frac{1}{2} z} W_{k, m}(z)=\frac{1}{2 \pi} \sum_{i,-i} \frac{1}{i} E\left(\frac{1}{2}+m, \frac{1}{2}-m, 1: 1-k: e^{i \pi} z\right), \\
H_{\nu}^{(1)}(z) H^{(\cdot)}(z)=2 \pi^{-5 / 2} \cos (\nu \pi) z^{-1} E\left(\frac{1}{2}+\nu, \frac{1}{2}-\nu, \frac{1}{2}:: z^{2}\right), \\
J_{\nu}(z) J_{-\nu}(z)=\{\Gamma(1-\nu) \Gamma(1+\nu)\}^{-1}{ }_{1} F_{2}\left(\frac{1}{2} ; 1-\nu, 1+\nu ;-z^{2}\right), \\
J_{\nu}^{2}(z)=\pi^{-1 / 2} z^{2 \nu} E\left(\frac{1}{2}+\nu: 1+\nu, 1+2 \nu: \frac{1}{z^{2}}\right),
\end{gathered}
$$

$S_{\mu, \nu}(z)=2^{\mu-1}\left\{\Gamma\left(\frac{1}{2}-\frac{1}{2} \mu-\frac{1}{2} \nu\right) \Gamma\left(\frac{1}{2}-\frac{1}{2} \mu+\frac{1}{2} \nu\right)\right\}^{-1}\left(\frac{z}{2}\right)^{\mu-1}$

$$
\begin{array}{r}
\times E\left(1, \frac{1}{2}-\frac{1}{2} \mu+\frac{1}{2} \nu, \frac{1}{2}-\frac{1}{2} \mu-\frac{1}{2} \nu:: \frac{1}{4} z^{2}\right), \\
M_{k, m}(i z) M_{k, m}(-i z)=z^{2 m+1_{2} F_{3}}\left(\begin{array}{l}
\frac{1}{2}+m+k, \frac{1}{2}, m, k ;-\frac{z^{2}}{4} \\
\frac{1}{2}+m, 1+m, 1+2 m
\end{array}\right),
\end{array}
$$

$z^{2 \mu} K_{2 v}\left(z e^{\frac{\pi i}{4}}\right) K_{2 v}\left(z e^{-\pi i / 4}\right)=2^{3 \mu-4} \pi^{-3 / 2}$

$$
\begin{aligned}
& \times \sum_{i,-i} \frac{1}{i} E\left(\frac{1}{2} \mu+\nu, \frac{1}{2} \mu-\nu, \frac{1}{2} \mu, \frac{1}{2} \mu+\frac{1}{2}, 1:: e^{i \pi} \frac{z^{4}}{64}\right), \\
& z^{\mu} K_{\nu}^{2}(z)=2^{-2} \pi^{1 / 2} \sum_{i,-i} \frac{1}{i} E\left(\nu+\frac{1}{2} \mu,-\nu+\frac{1}{2} \mu, \frac{1}{2} \mu, 1: \frac{1}{2} \mu+\frac{1}{2}: e^{i \pi} z^{2}\right), \\
& z^{\mu} K_{\nu}(z)=2^{\mu-2} \pi^{-1} \sum_{i,-i} \frac{1}{i} E\left(\frac{1}{2} \mu+\frac{1}{2} \nu, \frac{1}{2} \mu-\frac{1}{2} \nu, 1: e^{i \pi} \frac{z^{2}}{4}\right), \\
& {\left[e^{-\frac{\pi i}{4}} H_{a-b}^{(1)}\left(z^{1 / 2}\right) H_{a+b}^{(2)}\left(z^{1 / 2}\right)+e^{\frac{\pi i}{4}} H_{a+b}^{(1)}\left(z^{1 / 2}\right) H_{a-b}^{(2)}\left(z^{1 / 2}\right)\right]} \\
& =4 \pi^{-5 / 2} \cos (a \pi) \cos (b \pi) z^{-1 / 2} E\left(a+\frac{1}{2}, b+\frac{1}{2},-a+\frac{1}{2},-b+\frac{1}{2}: \frac{1}{2}: z\right), \\
& M_{k, m}(i z) M_{k, m}(-i z)=z^{2 m+1} F_{3}\left(\begin{array}{l}
\frac{1}{2}+m+k, \frac{1}{2}+m-k ;-\frac{z^{2}}{4} \\
\frac{1}{2}+m, 1+m, 1+2 m
\end{array}\right) \text {, } \\
& e^{-2 m \pi i}\{\Gamma(1+m+n) \Gamma(m-n)\}^{-1} Q_{n}^{m}\left[\left(1+z^{2}\right)^{1 / 2}\right] Q_{-n-1}^{m}\left[\left(1+z^{2}\right)^{1 / 2}\right] \\
& =\frac{\pi}{2 z}\left\{\Gamma\left(\frac{1}{2}-n\right) \Gamma\left(\frac{3}{2}+n\right)\right\}_{3}^{-1} F_{2}\left(\begin{array}{l}
\frac{1}{2}-m, \frac{1}{2}+m, \frac{1}{2} ;-\frac{1}{z^{2}} \\
\frac{1}{2}-n, \frac{3}{2}+n
\end{array}\right), \\
& H_{\nu}(z)-Y_{\nu}(z)=\pi^{-\nu-1} \cos \nu \pi z^{\nu-1} E\left(\frac{1}{2}, 1, \frac{1}{2}-\nu:: \frac{z^{2}}{4}\right), \\
& H_{b-a}^{(1)}\left(z^{1 / 2}\right) H_{b-a}^{(2)}\left(z^{1 / 2}\right)=2 \pi^{-5 / 2} \cos (b-a) \pi z^{-1 / 2} E\left(\frac{1}{2}, \frac{1}{2}+b-a, \frac{1}{2}+a-b:: z\right),
\end{aligned}
$$


$K_{\cdot(b-a)}\left(2^{3 / 2} z^{1 / 4} e^{\pi i / 4}\right) K_{2(b-a)}\left(2^{3 / 2} z^{1 / 4} e^{-\pi i / 4}\right)=2^{-4} \pi-\frac{3}{2} z^{-q}$

$$
\times \sum_{i,-i} \frac{1}{i} E\left(1, a, b, a+\frac{1}{2}, 2 a-b:: z e^{i \pi}\right)
$$

\section{Proof of the Main Theorem}

From (7), (8), and (9), we arrive at the formula

$$
\int_{0}^{\infty} z^{s-1} E\left(p: \alpha_{r}: q: \rho_{t}: z\right) d z=\frac{\Gamma(-s) \prod_{r=1}^{p} \Gamma\left(\alpha_{r}+s\right)}{\prod_{t=1}^{q} \Gamma\left(\rho_{t}+s\right)},
$$

where $R\left(\alpha_{r}+s\right)>0(r=1,2, \ldots, p)$ and $R(s)<0$.

We wish to solve the integral equation (1)

$$
g(x)=\int_{0}^{\infty} E\left(\begin{array}{l}
\nu-i x, \nu+i x, \alpha_{1}, \alpha_{2}, \ldots . ., \alpha_{p}: \frac{1}{y} \\
\beta_{1}, \beta_{2}, \ldots . ., \beta_{q}
\end{array}\right) f(y) d(y)
$$

where the conditions on the parameters and the function $f(y)$ are such that the integral is convergent.

Replace $x$ by $-i \xi$. Multiply both sides of (37) by $x^{-\xi} d \xi$ and integrate from $c-i \infty$ to $c+i \infty$, so getting

$$
\begin{aligned}
\frac{1}{2 \pi i} \int_{c-i x}^{c+i x} x^{-\xi} g(-i \xi) d \xi=\frac{1}{2 \pi i} \int_{c-i x}^{c+i x} x^{-\xi} \int_{0}^{\infty} E\left(\begin{array}{l}
\left.\nu-\xi, \nu+\xi, \alpha_{1}, \ldots, \alpha_{p}: \frac{1}{y}\right) f(y) d y d \xi \\
\beta_{1}, \ldots, \beta_{q}
\end{array}\right. \\
=\frac{-1}{4 \pi^{2}} \int_{0}^{\infty} f(y) d y \int_{c-i x}^{c+i x} x^{-\xi} d \xi \int_{\gamma-i x}^{\gamma+i x} \Gamma(\zeta) \Gamma(\nu+\xi-\zeta) \Gamma(\nu-\xi-\zeta) \frac{\prod_{l=1}^{\prime \prime} \Gamma\left(\alpha_{r}-\zeta\right)}{\prod_{t=1}^{q} \Gamma\left(\beta_{t}-\zeta\right)} y^{-\zeta} d \zeta,
\end{aligned}
$$

by $(9)$.

Now change the order of integration, so that the integral with respect to $\xi$ becomes the last and the last expression becomes

$$
\begin{gathered}
-\frac{1}{4 \pi^{2}} \int_{0}^{\infty} f(y) d y \int_{\gamma-i x}^{\gamma+i x} \Gamma(\zeta) \frac{\prod_{t=1}^{p} \Gamma\left(\alpha_{r}-\zeta\right)}{\prod_{t=1}^{q} \Gamma\left(\beta_{t}-\zeta\right)} y^{-\zeta} d \zeta \int_{c-i \infty}^{c+i x} \Gamma(\nu-\zeta-\xi) \Gamma(\nu-\zeta+\xi) x^{-\xi} d \xi \\
=-\frac{1}{4 \pi^{2}} \int_{0}^{\infty} f(y) d y \int_{\gamma_{-i x}}^{\gamma \cdot i x} \Gamma(\zeta) \frac{\prod_{t=1}^{p} \Gamma\left(\alpha_{r}-\zeta\right)}{\prod_{t=1}^{q} \Gamma\left(\beta_{t}-\zeta\right)} y^{-\zeta} x^{\nu-\zeta} d \zeta \times \int_{c-i x}^{c+i x} \Gamma(\xi) \Gamma(2 \nu-2 \zeta-\xi) x^{-\xi} d \xi \\
=-\frac{1}{4 \pi^{2}} \int_{0}^{\infty} f(y) d y \int_{\gamma-i x}^{\gamma+i x} \Gamma(\zeta) \frac{\prod_{\nu=1}^{p} \Gamma\left(\alpha_{r}-\zeta\right)}{\prod_{t=1}^{q} \Gamma\left(\beta_{t}-\zeta\right)} x^{\nu}(x y)^{-\zeta} d \zeta E\left(2 \nu-2 \zeta: \frac{1}{x}\right),
\end{gathered}
$$

by (9) again. 
Now apply (15) and the last expression becomes

$$
\begin{array}{r}
\frac{1}{2 \pi i} \int_{0}^{\infty} f(y) d y \int_{\gamma-i x}^{\gamma+i x} \Gamma(\zeta) \Gamma(\nu-\zeta) \Gamma\left(\nu+\frac{1}{2}-\zeta\right) 2^{2 \nu-2 \zeta-1} \frac{\prod_{r=1}^{p} \Gamma\left(\alpha_{r}-\zeta\right)}{\pi^{1 / 2} \prod_{t=1}^{q} \Gamma\left(\beta_{t}-\zeta\right)} x^{\nu}(1+x)^{-2 \nu+2 \zeta(x y)}-\zeta_{d \zeta} \\
=\frac{1}{2(\pi)^{1 / 2}}\left(\frac{4 x}{(1+x)^{2}}\right)^{\nu} \int_{0}^{\infty} E\left(\begin{array}{l}
\nu, \nu+\frac{1}{2}, \alpha_{1}, \ldots ., \alpha_{p}: \frac{(1+x)^{2^{-}}}{4 x y} \\
\beta_{1}, \ldots, \beta_{q}
\end{array}\right) f(y) d y
\end{array}
$$

by (9).

Now let $\bar{x}=\frac{4 x}{(1+x)^{2}}$ and

$$
\bar{g}(x)=\frac{1}{2 \pi i} \int_{c-i x}^{c+i x} x+\xi g(-i \xi) d \xi
$$

and the last expression becomes

$$
2(\pi)^{1 / 2} \bar{x}^{-\nu} \bar{g}(x)=\int_{0}^{\infty} E\left(\begin{array}{l}
\nu, \nu+\frac{1}{2}, \alpha_{1}, \ldots, \alpha_{p}: \frac{1}{x y} \\
\beta_{1}, \ldots ., \beta_{q}
\end{array}\right) f(y) d y
$$

Using the notation of ([7], p. 315), we have

$$
\mathscr{G}(s)=2(\pi)^{1 / 2} \int_{0}^{\infty} \bar{x}^{s-\nu-1} \bar{g}(x) d \bar{x}=2(\pi)^{1 / 24^{s-\nu}} \int_{0}^{-1}(1-x) x^{s-\nu-1}(1+x)^{2 \nu-2 s-1} \bar{g}(x) d x .
$$

Here write $1-s$ for $s$, apply (38) and get

$$
\begin{gathered}
\mathscr{G}(1-s)=\frac{4^{1-s-\nu}}{i(\pi)^{1 / 2}} \int_{c-i x}^{c+i x} g(-i \xi) d \xi \int_{0}^{-1}(1-x) x^{-s-\nu-\xi}(1+x)^{2 s+2 \nu-3} d x \\
=\frac{e^{i \pi(-s-\nu)}}{i(\pi)^{1 / 2}} \frac{\Gamma(2 s+2 \nu-2)}{4^{s+\nu-1}} \int_{c-i x}^{c+i x}-g(-i \xi)\left[\frac{\Gamma(1-s-\nu-\xi)}{\Gamma(s+\nu-\xi-1)}+\frac{\Gamma(2-s-\nu-\xi)}{\Gamma(s+\nu-\xi)}\right] e^{i \pi \xi} d \xi \\
=\frac{e^{-i \pi(s+\nu)}}{i \pi} \Gamma\left(s+\nu-\frac{1}{2}\right) \Gamma(s+\nu-1) \int_{c-i x}^{c+i x} e^{-\pi i \xi} \xi g(-i \xi) \frac{\Gamma(1-s-\nu-\xi)}{\Gamma(s+\nu-\xi)} d \xi .
\end{gathered}
$$

Thus

$$
\mathscr{G}(1-s)=\frac{e^{-i \pi(s+\nu)}}{i \pi} \Gamma\left(s+\nu-\frac{1}{2}\right) \Gamma(s+\nu-1) \int_{c-i \infty}^{c+i \infty} e^{-i \pi \xi} \xi g(-i \xi) \frac{\Gamma(1-s-\nu-\xi)}{\Gamma(s+\nu-\xi)} d \xi .
$$

Also from (36) and (39)

$$
R(1-s)=\Gamma(1-s) \Gamma(\nu-1+s) \Gamma\left(v-\frac{1}{2}+s\right) \frac{\prod_{r=1}^{p} \Gamma\left(\alpha_{r}-1+s\right)}{\prod_{t=1}^{q} \Gamma\left(\beta_{t}-1+s\right)},
$$

and so by [7], p. 316,

$$
f(x)=\frac{1}{2 \pi i} \int_{c^{\prime}-i \infty}^{c^{\prime}+i \infty} \frac{\mathscr{G}(1-s)}{\mathscr{R}(1-s)} x^{-s} d s=\frac{1}{2(\pi i)^{2}} \int_{c^{\prime}-i \infty}^{c^{\prime}+i_{\infty}} x^{-s} d s \int_{c-i \infty}^{c+i \infty} e^{-\pi i(s+\nu+\xi)} \xi g(-i \xi)
$$




$$
\begin{gathered}
\times \frac{\Gamma(1-s-\nu-\xi) \prod_{t=1}^{q} \Gamma\left(\beta_{t}-1+s\right)}{\Gamma(s+\nu-\xi) \Gamma(1-s) \prod_{r=1}^{p} \beta\left(\alpha_{r}-1+s\right)} d \xi=\frac{1}{\pi i} \int_{c-i \infty}^{c+i \infty} e^{-\pi i(\nu+\xi)} \xi g(-i \xi) d \xi \\
\times \frac{1}{2 \pi i} \int_{c^{-i \infty}}^{c+i \infty} \frac{\Gamma(1-\nu-\xi+s) \prod_{t=1}^{q} \Gamma\left(\beta_{t}-1-s\right)}{\Gamma(\nu-\xi-s) \Gamma(1+s) \prod_{r=1}^{p} \Gamma\left(\alpha_{r}-1-s\right)}\left(e^{i \pi} x\right)^{s} d s,
\end{gathered}
$$

using the definition of the generalized $E$-function (see [1], p. 419) namely

$$
\begin{aligned}
& E\left(\begin{array}{l|l}
p ; & \alpha_{r} \mid m ; \rho_{q+s}: x \\
q ; & \rho_{s} \\
l+1, \alpha_{p+r}, 1
\end{array}\right)=\frac{1}{2 \pi i} \int_{c-i \infty}^{c+i \infty} \frac{\prod_{r=1}^{p} \Gamma\left(\alpha_{r}-\zeta\right) \prod_{s=1}^{m} \Gamma\left(\zeta-\rho_{q+s}+1\right)}{\prod_{s=1}^{q} \Gamma\left(\rho_{s}-\zeta\right) \prod_{r=1}^{l} \Gamma\left(\zeta-\alpha_{p+r}+1\right)} x^{\zeta} d \zeta \\
& =\pi^{m-l-1} \sum_{s=1}^{m} \frac{\prod_{r=1}^{l} \sin \left(\rho_{q+s}-\alpha_{p+r}\right) \pi}{\prod_{s=1}^{m} \sin \left(\rho_{q+s}-\rho_{q+t}\right) \pi} x^{\rho_{q+s^{-1}}} \times E\left(\begin{array}{l}
p+l ; \alpha_{r}-\rho_{q+s}+1: \omega x \\
\rho_{1}-\rho_{q+s}+1, \ldots * \ldots, \rho_{q+m}-\rho_{q+s}+1
\end{array}\right),
\end{aligned}
$$

where $\omega=e^{ \pm i \pi}$ or 1 according as $l+m$ is even or odd; the expression (A) becomes

$$
\frac{-x^{\nu-1}}{i(\pi)^{2}} \int_{c-i \infty}^{c+i \infty} \xi g(-i \xi) x^{\xi} \sin (\nu+\xi) \pi E\left(\begin{array}{l}
1-\nu-\xi, \beta_{1}-\nu-\xi, \ldots, \beta_{t}-\nu-\xi: x \\
1-2 \xi, \alpha_{1}-\nu-\xi, \ldots, \alpha_{p}-\nu-\xi
\end{array}\right) d \xi
$$

Here put $c=0$, note that $g(x)=g(-x)$, so getting

$f(x)=\frac{x^{\nu-1}}{i(\pi)^{2}} \int_{0}^{\infty} y g(y)\left[\frac{1}{i} \sum_{i,-i} i x^{i y} \sin (i \pi y+\nu \pi) E\left(\begin{array}{l}1-\nu-i y, \beta_{1}-\nu-i y, \ldots ., \beta_{q}-\nu-i y: x \\ 1-2 i y, \alpha_{1}-\nu-i y, \ldots ., \alpha_{p}-\nu-i y\end{array}\right)\right] d y$

With (43) established, we have the transform pair (1) and (2).

\section{Derivation of the Kantorovich-Lebedev and Generalized Mehler Transforms}

In the transform pair (1) and (2) take $p=q=0, \nu=\frac{1}{2}-k$, apply (18) and (13), so getting the transform pair

$$
\begin{gathered}
g(x)=\Gamma\left(\frac{1}{2}-k-i x\right) \Gamma\left(\frac{1}{2}-k+i x\right) \int_{0}^{\infty} e^{1 / 2 y} y^{k} W_{k, i x}\left(\frac{1}{y}\right) f(y) d y \\
f(x)=\frac{x^{-k-1 / 2}}{i(\pi)^{2}} e^{-1 / x} \int_{0}^{\infty} y g(y)\left[\frac{1}{i} \sum_{i,-i} i x^{i y} \sin \left(\frac{1}{2}-k+i y\right) \pi \frac{\Gamma\left(\frac{1}{2}+k-i y\right)}{\Gamma(1-2 i y)}{ }_{1} F_{1}\left(\begin{array}{l}
\frac{1}{2}-k-i y ; \frac{1}{x} \\
1-2 i y
\end{array}\right)\right] d y,
\end{gathered}
$$

where we have used the Kummer transformation

$$
{ }_{1} F_{1}\left(\begin{array}{l}
\alpha ; x \\
\rho
\end{array}\right)=e^{x}{ }_{1} F_{1}\left(\begin{array}{l}
\rho-\alpha ;-x \\
\rho
\end{array}\right)
$$


Now apply the relations ([1], p. 351 and p. 352)

$$
\begin{gathered}
{ }_{1} F_{1}\left(\frac{1}{2}-k-i y ; 1-2 i y ; x\right)=x^{-1 / 2+i y} e^{\frac{x}{2}} M_{k,-i y}(x), \\
W_{k, i y}(x)=\sum_{y,-y} \frac{\Gamma(-2 i y)}{\Gamma\left(\frac{1}{2}-k-i y\right)} M_{k, i y}(x) ;
\end{gathered}
$$

and so get the transform pair

$$
\begin{gathered}
g(x)=\Gamma\left(\frac{1}{2}-k-i x\right) \Gamma\left(\frac{1}{2}-k+i x\right) \int_{0}^{\infty} W_{k, i x}\left(\frac{1}{y}\right) e^{1 / 2 y} y^{k} f(y) d y \\
f(x)=x^{-k} e^{-1 / 2 x} \frac{1}{i(\pi)^{2}} \int_{0}^{\infty} y g(y) W_{k, i y}\left(\frac{1}{x}\right) \sin (2 i y) \pi d y
\end{gathered}
$$

In (49) replace $y$ by $\frac{1}{y}$, in (50), $x$ by $\frac{1}{x}$ and then replace $e^{y / 2} y^{-k} f\left(\frac{1}{y}\right)$ by $f(y)$. The transform pair is

$$
\begin{gathered}
g(x)=\Gamma\left(\frac{1}{2}-k-i x\right) \Gamma\left(\frac{1}{2}-k+i x\right) \int_{0}^{\infty} W_{k, i x}(y) f(y) d y, \\
f(x)=\frac{1}{(x \pi)^{2}} \int_{0}^{\infty} y \sinh (2 y \pi) W_{k, i y}(y) g(y) d y .
\end{gathered}
$$

In (51), (52) put $k=0$, apply the relation

$$
W_{0, m}(x)=\left(\frac{x}{\pi}\right)^{1 / 2} K_{m}\left(\frac{x}{2}\right)
$$

\begin{tabular}{|c|c|}
\hline$g(x)$ & $f(x)=\int_{0}^{x} K_{i y}(x) g(y) d y$ \\
\hline $\begin{array}{l}\cosh (\alpha x) \cos (z x) \\
\qquad|\operatorname{Im} \alpha|+|\operatorname{Im} z|<\frac{\pi}{2}\end{array}$ & $\frac{\pi}{2} e^{-x \cosh z \cos \alpha} \cos (x \sinh z \sin \alpha)$ \\
\hline $\begin{array}{l}\sinh (\alpha x) \sin (z x) \\
\qquad|\operatorname{Im} \alpha|+|\operatorname{Im} z|<\frac{\pi}{2}\end{array}$ & $\frac{\pi}{2} e^{-x \cosh z \cos \alpha} \sin (x \sinh z \sin \alpha)$ \\
\hline $\begin{array}{c}\cosh \left(\frac{\pi}{2} x\right) \cos (z x) \\
|\operatorname{Im} z|<\frac{\pi}{2}\end{array}$ & $\frac{\pi}{2} \cos (x \sinh z)$ \\
\hline
\end{tabular}

and so obtain the Kantorovich-Lebedev transform (3) and (4). For a study how such transforms arise from second order differential equations, see [8] and [9].

In the following table, we give a short list of integrals corresponding to formula (4).

TABLE 1. Kantorovich-Lebedev transforms 


\begin{tabular}{|c|c|}
\hline$g(x)$ & $f(x)=\int_{0}^{\infty} K_{i y}(x) g(y) d y$ \\
\hline $\begin{array}{c}\sinh \left(\frac{\pi}{2} x\right) \sin (z x) \\
|\operatorname{Im} z|<\frac{\pi}{2}\end{array}$ & $\frac{\pi}{2} \sin (x \sinh z)$ \\
\hline $\begin{array}{c}\cosh \left(\frac{\pi}{4} x\right) \cos (z x) \\
|\operatorname{Im} z|<\frac{\pi}{2}\end{array}$ & $\frac{\pi}{2} e^{-\frac{x}{\sqrt{2}} \cosh z} \cos \left(\frac{x}{\sqrt{2}} \sinh z\right)$ \\
\hline $\begin{array}{c}x \sinh (\pi x) E\left(\begin{array}{l}\left.\frac{k+i x}{2}, \frac{k-i x}{2}, \alpha_{1}, \ldots ., \alpha_{p}: \frac{z}{4}\right) \\
\rho_{1}, \ldots . \rho_{q}\end{array}\right. \\
|\arg z|<\pi\end{array}$ & $\frac{\pi^{2}}{2^{k-1}} x^{k} E\left(p ; \alpha_{r}: q ; \rho_{s}: \frac{z}{x^{2}}\right)$ \\
\hline $\begin{array}{c}x \sinh \left(\frac{1}{2} \pi x\right) K_{i x / 2}\left(\frac{z}{8}\right) \\
|\arg z|<\frac{\pi}{2}\end{array}$ & $\pi^{3 / 2} z^{-1 / 2} \exp \left(-\frac{z}{8}-\frac{x^{2}}{z}\right)$ \\
\hline $\begin{array}{c}x \sinh (\pi x) W_{k, i x / 2}\left(\frac{z}{4}\right) \times \Gamma\left(\frac{1}{2}-k-\frac{i x}{2}\right) \Gamma\left(\frac{1}{2}-k-\frac{i x}{2}\right) \\
R\left(\frac{1}{2}-k\right)>0,|\arg z|<\frac{\pi}{2}\end{array}$ & $2^{2 k} \pi^{2}\left(\frac{x z}{4}\right)^{1-2 k} \exp \left(-\frac{z}{8}-\frac{x^{2}}{z}\right)$ \\
\hline$x \sinh (\pi x) \Gamma\left(\frac{k+i x}{2}\right) \Gamma\left(\frac{k-i x}{2}\right)$ & $\frac{\pi^{2}}{2^{k-1}} \Gamma(1+\nu) x^{k}\left(\frac{z}{x^{2}}\right) \frac{\nu}{2} J_{\nu}\left(\frac{2 x}{\sqrt{z}}\right)$ \\
\hline $\begin{array}{l}\times{ }_{2} F_{1}\left(\frac{k+i x}{2}, \frac{k-i x}{2} ; 1+\nu ;-\frac{4}{z}\right) \\
R(k)>0 \quad z \text { is real and positive }\end{array}$ & \\
\hline $\begin{array}{c}x \sinh (\pi x) E\left(\frac{k+i x}{2}, \frac{k-i x}{2}, \alpha: \frac{z}{4}\right) \\
z \text { is real and positive }\end{array}$ & $x^{k} \Gamma(\alpha)\left(1+\frac{x^{2}}{z}\right)^{-\alpha}$ \\
\hline $\begin{array}{c}x \sinh (\pi x) \Gamma(m+i x) \Gamma(m-i x) \times \begin{cases}P_{i x}^{1 / 2-1 / 2}(z) & |z|>1 \\
T_{i, 2-1 / 2}^{1 / 2-1 / 2}(z), & |z|<1\end{cases} \\
R(m)>0, R(z)>-1 .\end{array}$ & $\frac{\pi^{3 / 2}}{2^{1 / 2}}\left(z^{2}-1\right)^{1 / \frac{m}{2}-\frac{1}{4}} x^{\prime \prime \prime} e^{-x z}$ \\
\hline$x \tanh (\pi x) P_{i x-1 / 2}(z)$ & $\left(\frac{1}{2} \pi x\right)^{1 / 2} e^{-x z}$ \\
\hline $\begin{array}{l}x \tanh (\pi x) P_{i x-1 / 2}(z) K_{i x}(\alpha) \\
\qquad|\arg \alpha|<\frac{\pi}{2},|\arg (z-1)|<\pi\end{array}$ & $\frac{\pi}{2}(\alpha x)^{1 / 2}\left(x^{2}+\alpha^{2}+2 \alpha z x\right)^{-1 / 2} \exp \left[-\left(x^{2}+\alpha^{2}+2 \alpha z x\right)^{1 / 2}\right]$ \\
\hline $\begin{array}{l}x \sinh (\pi x) E\left(\frac{1}{2}+m+i x, \frac{1}{2}+m-i x, l: m+1: 2 z\right) \\
\quad|\arg z|<\frac{\pi}{2}\end{array}$ & $\pi^{3 / 2} 2^{m-1 / 2} \Gamma(l) z^{l} e^{-x} x^{m+1 / 2}(z+x)^{-l}$ \\
\hline
\end{tabular}




$$
x \tanh (\pi x) K_{i x}(z)
$$$$
x \sinh (\pi x) E\left(\begin{array}{l}
\lambda+i x, \lambda-i x, \alpha_{1}, \ldots, \alpha_{p}: z \\
\lambda+\frac{1}{2}, \rho_{1}, \ldots ., \rho_{q}
\end{array}\right)
$$

$$
R(\lambda)>0,|\arg z|<\frac{\pi}{2}
$$

$$
\begin{gathered}
x \sinh (\pi x) K_{2 i x}(\alpha) \\
|\arg \alpha|<\frac{\pi}{4}
\end{gathered}
$$

$$
\begin{gathered}
x \sinh (\pi x) \Gamma\left(\frac{1}{2}-k+i x\right) \Gamma\left(\frac{1}{2}-k-i x\right) \times W_{k, i x}(z) \\
R\left(\frac{1}{2}-k\right)>0,|\arg z|<\frac{\pi}{2}
\end{gathered}
$$$$
x \sinh (\pi x) E\left(\begin{array}{l}
\lambda+i x, \lambda-i x, \frac{1}{2}+n, \frac{1}{2}-n: 4 z \\
\lambda+\frac{1}{2}
\end{array}\right)
$$

$$
R\left(\lambda>0,|\arg z|<\frac{\pi}{2}\right.
$$

$$
x \sinh (\pi x) E\left(\begin{array}{l}
\lambda+i x, \lambda-i x, \alpha: z \\
\lambda+\frac{1}{2}
\end{array}\right)
$$

$$
R(\lambda)>0,|\arg z|<\frac{\pi}{2}
$$

$$
x \sinh (\pi x) E\left(\begin{array}{l}
\lambda+i x, \lambda-i x, \frac{1}{2}-k+m, \frac{1}{2}-k-m: z \\
\lambda+\frac{1}{2}
\end{array}\right)
$$

$$
R(\lambda)>0,|\arg z|<\frac{\pi}{2}
$$

$$
x \sinh (\pi x) \Gamma(\lambda+i x) \Gamma(\lambda-i x)_{2} F_{2}\left(\begin{array}{l}
\lambda+i x, \lambda-i x ;-\frac{1}{z} \\
\lambda+\frac{1}{2}, \nu+1
\end{array}\right)
$$

$$
R(\lambda)>0, z \text { is real and positive }
$$

$x \sinh (\pi x) \Gamma\left(\frac{1}{2}+m+i x\right) \times \Gamma\left(\frac{1}{2}+m-i x\right) M_{i x, m}(i z) M_{i x, m}(-i z)$

$$
R(m)>-\frac{1}{2} \text { and } z \text { is real and positive }
$$

$x \sinh (\pi x) K_{2 i x}(\alpha) K_{i x}(\beta)$

$$
2|\arg \alpha|+|\arg \beta|<\pi
$$

$$
\begin{aligned}
& \frac{1}{2} \pi(z x)^{1 / 2}(z+x)^{-1} \exp (-z-x) \\
& 2^{\lambda-1} \pi^{3 / 2} x^{\lambda} e^{-x} E\left(p ; \alpha_{r}: q ; \rho_{s}: \frac{z}{2 x}\right)
\end{aligned}
$$$$
\frac{\pi^{3 / 2} \alpha}{2^{7 / 2} x^{1 / 2}} \exp \left(-x-\frac{\alpha^{2}}{8 x}\right)
$$$$
2^{-k-1 / 2} \pi^{3 / 2} \Gamma(1-k) x^{1 / 2-k} z^{k}\left[\exp \left(-x-\frac{1}{2} z\right)\right]\left(1+\frac{2 x}{z}\right)^{-1+k}
$$

$2^{\lambda-1 / 2} \pi^{2} \sec (n \pi) x^{\lambda-1 / 2} z^{1 / 2} \exp \left(-x+\frac{z}{x}\right) K_{n}\left(\frac{z}{x}\right)$

$$
2^{\lambda-1} \pi^{3 / 2} x^{\lambda} e^{-x} \Gamma(\alpha)\left(1+\frac{2 x}{z}\right)^{-\alpha}
$$

$2^{\lambda+k-1} \pi^{3 / 2} \Gamma\left(\frac{1}{2}-k+m\right) \Gamma\left(\frac{1}{2}-k-m\right) \Gamma\left(\frac{1}{2}-m\right)$

$$
\times z^{-k} x^{\lambda+k}\left[\exp \left(-x+\frac{z}{4 x}\right)\right] W_{k, m}\left(\frac{z}{2 x}\right)
$$

$2^{\lambda-\frac{\nu}{2}-1} \pi^{3 / 2} x^{\lambda-1 / \frac{\nu}{2}} z^{1 / \frac{L}{2}} \Gamma\left(\lambda+\frac{1}{2}\right) \Gamma(1+\nu) J_{\nu}\left[\left(\frac{8 x}{z}\right)^{1 / 2}\right]$

$2^{m-1 / 2} \pi^{3 / 2} x^{1 / 2+m} z^{-1-2 m_{0}} F_{2}\left(: \frac{1}{2}+m, 1+m ;-\frac{x z^{2}}{2}\right)$

$$
\frac{\pi^{3 / 2} \alpha}{16(\beta x)^{1 / 2}}\left(\frac{4 \beta x}{4 \beta x+\alpha^{2}}\right)^{1 / 2} \exp \left[-(\beta+x)\left(\frac{4 \beta x+\alpha^{2}}{4 \beta x}\right)^{1 / 2}\right]
$$


To derive the generalized Mehler transform pair (5) and (6) take in (1), (2), $p=0, q=1, \nu=\frac{1}{2}$ with $\beta_{1}=1-k$, so getting

$$
\begin{aligned}
& \text { with } \beta_{1}=1-k \text {, so getting } g(x)=\frac{\pi}{\Gamma(1-k) \cosh (\pi x)} \int_{0}^{\infty} F_{1}\left(\begin{array}{l}
\frac{1}{2}-i x, \frac{1}{2}+i x ;-y \\
1-k
\end{array}\right) f(y) d y, \\
& f(x)=\frac{x^{-1 / 2}}{i \pi^{2}} \int_{0}^{\infty} y g(y)\left[\frac{1}{i} \sum_{i,-i} i x^{i y} \sin \left(\frac{\pi}{2}+i \pi y\right) \frac{\Gamma\left(\frac{1}{2}-i y\right) \Gamma\left(\frac{1}{2}-k-i y\right)}{\Gamma(1-2 i y)}\right. \\
& \left.\times{ }_{2} F_{1}\left(\begin{array}{l}
\left.\left.\frac{1}{2}-i y, \frac{1}{2}-k-i y ;-\frac{1}{x}\right)\right] d y . \\
1-2 i y
\end{array}\right)\right]
\end{aligned}
$$

Now write $\frac{1}{2}(y-1)$ for $y$ in (54) and $\frac{1}{2}(x-1)$ for $x$ in (55) and get

$$
g(x)=\frac{\pi}{2 \Gamma(1-k) \cosh \pi x} \int_{1}^{\infty}{ }_{2} F_{1}\left(\begin{array}{l}
\frac{1}{2}-i x, \frac{1}{2}+i x ; \frac{1-y}{2} \\
1-k
\end{array}\right) f\left(\frac{y-1}{2}\right) d y,
$$

$f\left(\frac{x-1}{2}\right)=\frac{1}{i \pi^{2}}\left(\frac{x-1}{2}\right)^{-1 / 2} \int_{0}^{x} y g(y)\left[\frac{1}{i} \sum_{i,-i}\left(\frac{x-1}{2}\right)^{i, y} \sin \left(\frac{\pi}{2}+i \pi y\right)\right.$

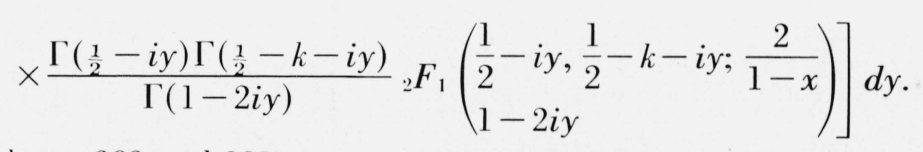

If we use the relationships ([1], pp. 303 and 391)

$$
P_{n}^{-m}(z)=\frac{1}{\Gamma(m+1)}\left(\frac{z-1}{z+1}\right)^{\frac{1}{2} m}{ }_{2} F_{1}\left(\begin{array}{l}
-n, n+1 ; \frac{1-z}{2} \\
m+1
\end{array}\right)
$$

$$
\begin{aligned}
\Gamma\left(\frac{1}{2}\right)\left(z^{2}-1\right)^{-\frac{1}{2} m} P_{n}^{-m}(z)=\frac{2^{n} \Gamma\left(n+\frac{1}{2}\right)}{\Gamma(n+m+1)}(z-1)^{n-m_{2} F_{1}}\left(\begin{array}{l}
-n, m-n ; \frac{2}{z-1} \\
-2 n
\end{array}\right) \\
\quad+\frac{2^{-n-1} \Gamma\left(-n-\frac{1}{2}\right)}{\Gamma(m-n)}(z-1)^{-n-m-1_{2} F_{1}}\left(\begin{array}{l}
n+1, n+m+1 ; \frac{2}{z-1} \\
2 n+2
\end{array}\right),
\end{aligned}
$$

in (56) and (57) respectively we obtain the generalized Mehler transform pair (5) and (6). A short list of integrals corresponding to formula (6) is given in the following table:

TABLE 2. Generalized Mehler transforms

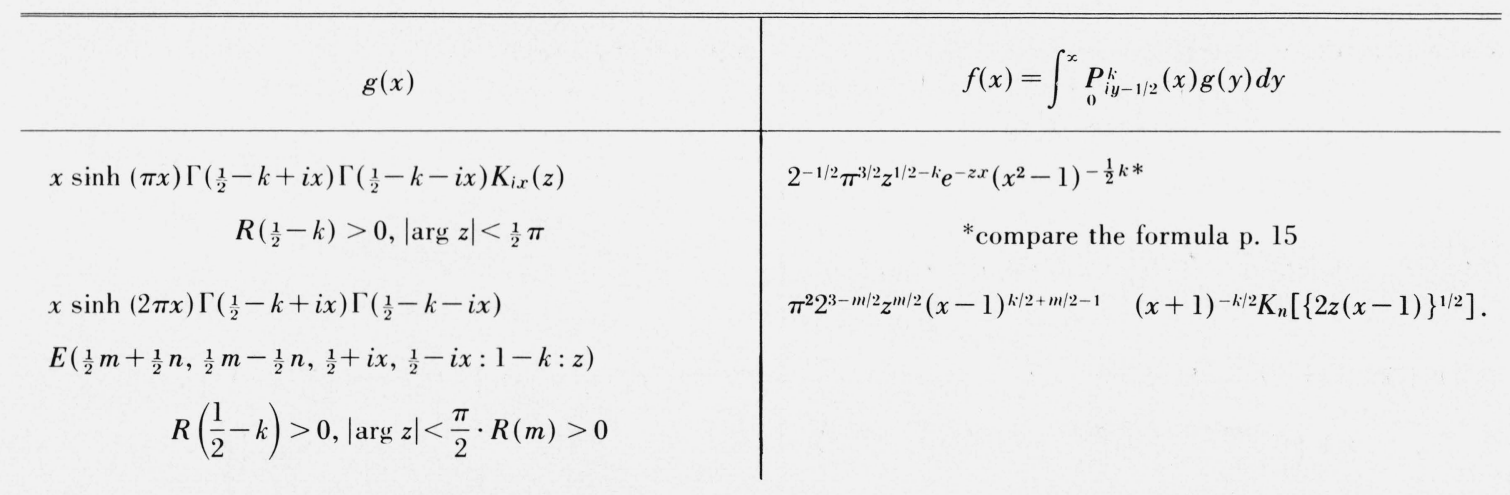




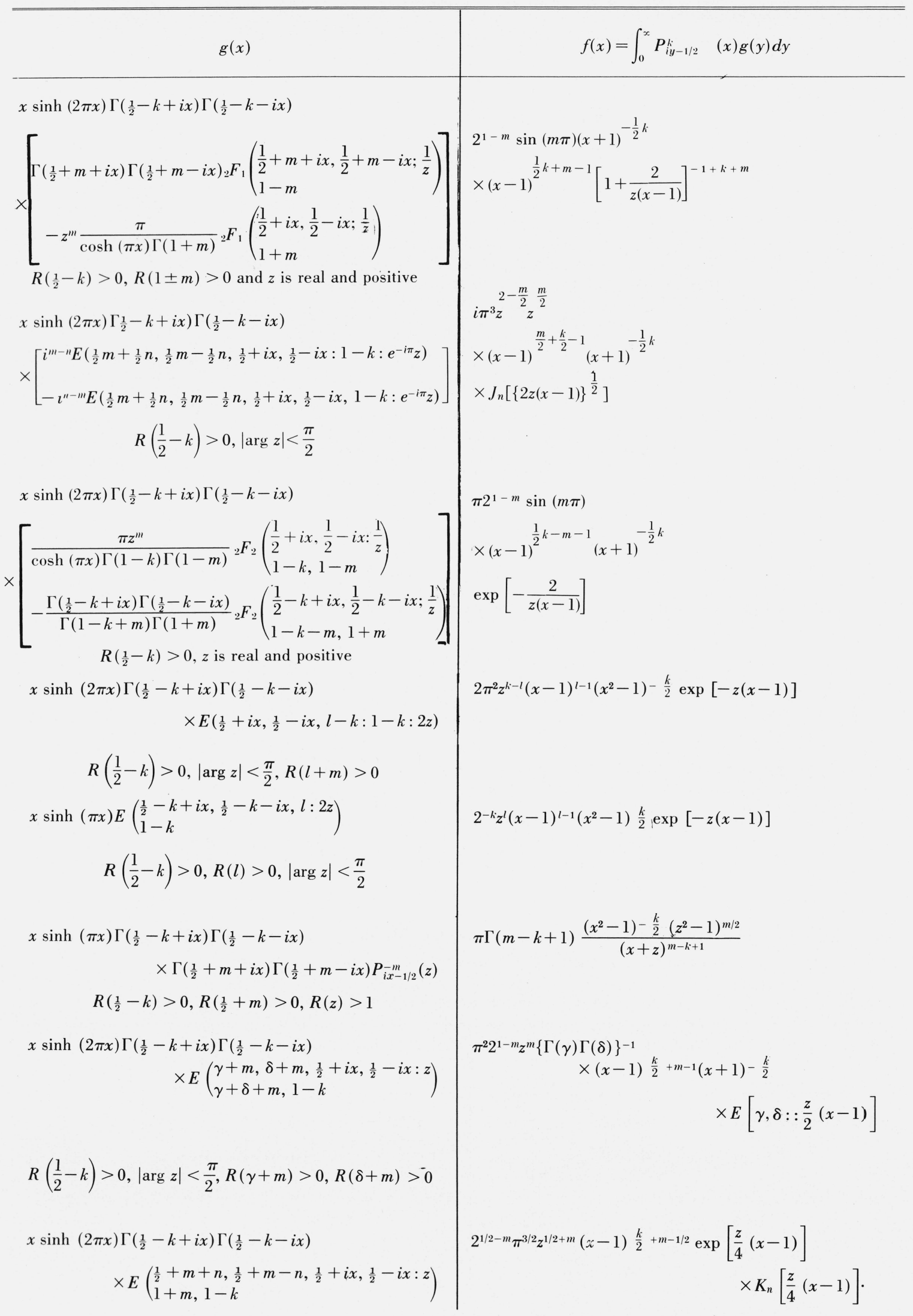


TABLE 2. Generalized Mehler transforms-Continued

$$
\begin{aligned}
& R\left(\frac{1}{2}-k\right)>0,|\arg z|<\frac{\pi}{2} \\
& \left.x \sinh (2 \pi x) \Gamma\left(\frac{1}{2}-k+i x\right) \Gamma \cdot \frac{1}{2}-k-i x\right) \\
& \times E\left(\begin{array}{l}
\frac{1}{2}-k^{\prime}+m^{\prime}+m, \frac{1}{2}-k^{\prime}-m^{\prime}+m, \frac{1}{2}+i x, \frac{1}{2}-i x: z \\
1-2 k^{\prime}+m, 1-k
\end{array}\right) \\
& R\left(\frac{1}{2}-k\right)>0,|\arg z|<\frac{\pi}{2} \\
& \begin{aligned}
& \pi^{2} 2^{1+k^{\prime}-m} z^{m-k^{\prime}}(x-1)^{-\frac{1}{2} k+m-k^{\prime}-1}(x+1)^{-\frac{k}{2}} \\
& \times \times \exp \left[\frac{z}{4}(x-1)\right] W_{k^{\prime}, m^{\prime}}\left[\frac{z}{2}(x-1)\right]
\end{aligned}
\end{aligned}
$$

Other applications can be obtained from (1) and (2) by special choices of the parameters. Thus (1) and (2) in combination with (25) yield the transform pair

$$
\begin{gathered}
g(x)=\int_{0}^{\infty} S_{2 \mu, 2 i x}(y) f(y) d y, \\
f(x)=-\frac{2^{2 \mu-2}}{i \pi x} \int_{0}^{\infty} y g(y)\left[\frac{\Gamma\left(\frac{1}{2}-\mu-i y\right)}{\Gamma\left(\frac{1}{2}+\mu-i y\right)} J_{-2 i y}(x)-\frac{\Gamma\left(\frac{1}{2}-\mu+i y\right)}{\Gamma\left(\frac{1}{2}+\mu+i y\right)} J_{2 i y}(x)\right] d y,
\end{gathered}
$$

which may be called $S$-transforms.

When $p=0, q=1$ then the $E$-functions in (1) and (2) reduce to the ordinary hypergeometric functions of Gauss and the following transform pair is obtained:

$$
\begin{gathered}
g(x)=\int_{0}^{\infty}{ }_{2} F_{1}\left(\begin{array}{l}
\nu-i x, \nu+i x ;-y \\
\beta
\end{array}\right) f(y) d y, \\
f(x)=\frac{\Gamma(\beta) x^{\nu-1}}{i \pi^{2}} \int_{0}^{\infty} \Gamma(\nu-i y) \Gamma(\nu+i y) y g(y) \\
\times\left[\frac{1}{i} \sum_{i,-i} i x^{i y} \frac{\sin (i \pi y+\nu \pi) \Gamma(1-\nu-i y) \Gamma(\beta-\nu-i y)}{\Gamma(1-2 i y)}{ }_{2} F_{1}\left(\begin{array}{l}
1-\nu-i y, \beta-\nu-i y ;-\frac{1}{x} \\
1-2 i y
\end{array}\right)\right] d y .
\end{gathered}
$$

\section{References}

[1] MacRobert, T. M., Functions Of A Complex Variable, 5th edition, London (1962).

[2] Erdelyi, A., Magnus, W., Oberhettinger, F., and Tricomi, F., Tables Of Integral Transforms (McGraw-Hill, Book Co., New York, N.Y., 1954).

[3] Kantorovich, M. and Lebedev, N., On a method of solution of some problems in diffraction theory, Journal Physics, Moscow 1 (1939).

[4] Wimp, J., A class of integral transforms, Proc. Edin. Math. Soc. 14, series II (1964).

[5] Lownders, J., Note on the generalized Mehler transform, Proc. Cambr. Philos. Soc. 60 (1964).

[6] Oberhettinger, F. and Higgins, T., Tables of Lebedev, Mehler and the generalized Mehler transforms, Boeing Scientific Research Laboratories, Mathematical notes, 246, Washington, 1961.

[7] Titchmarsh, E. C., An Introduction to the Theory of Fourier Integral, Oxford (1937).

[8] Mercer, M., Integral transform pairs arising from second order differential equations, Proc. Edin. Math. Soc. (2), (13), pp. 63-68 (1962).

[9] Titchmarsh, E. C., Eigenfunctions Expansions Associated With Second Order Differential Equations, 2d edition, Oxford (1962).

(Paper 71B2 \& 3-203) 